\title{
A TEST OF INTERVAL EQUIVALENCE
}

\author{
David Willer \\ University of Kansas.
}

Computer applications of research findings usually assume either a metric or interval level of measurement. This assumption, not always consciously made, follows necessarily as a consequence of the mathematical manipulations which are performed. According to the classical theory of measurement as advanced by Campbel1 ${ }^{1}$, metric measurement is determined by a process of physical addition. This addition is direct in the case of fundamental measurement and indirect for derived measurement. Physical addition is, in turn, dependent upon the possibility of performing a join, the physical equivalent of addition. Thus, a length, ac, if it can be produced by joining lengths $a b$ and $b c$, may be thought of as equal to their summation. Similarily, if two masses, $x$ and $y$, balance a mass $z$, then their arithmetic sum may be considered equivalent to $z$. (The $x, y$ join in this case is completed by placing the two masses, $x$ and $y$, on the same pan.)

The possibilities of performing joins in social science measurement appear limited. Indeed, when the idea of a join is most strictly interpreted, there is apparently only one obviously join-based quantity unique to the social sciences--money. If unable to perform joins to produce quantities, our measurements could be limited, as has been suggested by Coleman, 2 to the counting of such objects as people or social relationships, or we could accept the manipulative limits of comparative ordinal measurement.

Hempe ${ }^{3}$ and Dingle ${ }^{4}$ have questioned the necessity for such a restrictive basis for measurement. Hempel maintained that a quantity is not determined by a set of physical operations but by a conceptual construct, while Dingle proposed that the scope of possible operations should be widened beyond the notion of a join to include any operation which yields a number. In both cases empirical import, the level and generality of mathematical relationship between the property measured and other quantities, was the primary criterion of measurement; and the necessity of a physical join was completely dispensed with. If a physical join is not necessary for the measurement of a quantity then the possibility arises that metric and interval levels of relationship may be established by non-join methods presently used, such as the Thurstone and Guttman methods; but the problem remains of determining if or when these methods or any other methods yield scales which may be considered interval or metric.

In classical metric measurement, once a means of determining equivalence and performing a join has been established, an arbitrary unit can be established, any number of which could then be generated by the means for determining equivalences. Collections of these unit standards could

$$
\text { K.J.S., Vol. III, No. } 395 \quad \text { Summer, } 1967
$$


consequently be joined and placed equivalent to any object possessing the property to be measured. In this way the measurement of the quantity of the property of that object is equivalent to counting the number of unit standards necessary for equivalence. The purpose of such a join is to reduce measurement to counting. The avowed purpose of the process as a whole is to establish a strict isomorphism between the operations of measurement and the operations of arithmetic. Arithmetic manipulations could then find their exact counterparts in measurement (and presumably in reality) if and only if the metric level was reached.

Such strict isomorphism is not desired just for its own sake but so that mathematical manipulations upon the values given by the measurement will not result in error. Just as the need for a join was demonstrated to be excessively restrictive, the emphasis upon physical-mathematical isomorphism now seems misplaced. Instoud, the emphasis should be placed on whether or not the resu...ts of a measure may be subjected to certain mathematical manipulations without creating significant error. Only this criterion is open to direct determination. A strict mathematical-physical isomorphism may only appear to be present or absent, while the extent of error created through mathematical manipulation can be determined if a reasonable level of empirical import is given. Following is a special case of determination of error through manipulation.

Given are two variables, $x$ and $y$, neither otherwise known to be of interval level. Given also is the knowledge that $\mathrm{x}$ and $\mathrm{y}$ may be related linearly such that

$$
x=y+e
$$

where e, an error term, has either a small value relative to $x$ and $y$ or is zero. Assume for the moment that for this relationship both a Pearson $r$ and a Spearman $p$ (rank correlation coefficient, or rho) are computed. If all the ass:ptions of both of the tests are met, the resulting correlational values can be related as in the following equation.

$$
r_{x y}=2 \sin \left(\frac{\pi}{6} p_{x y}\right)
$$

As long as the assimptions of both tests are met the values of both sides of the equation will be dependent only upon the size of the error $e$ and will therefore adequately reflect the level of relationship found and the equality will hold. If, however, either $\mathrm{x}$ or $\mathrm{y}$ or both are not either metric or interval then:

$$
\mathrm{r}_{\mathrm{xy}}<2 \sin \left(\frac{\pi}{6} \mathrm{p}_{\mathrm{xy}}\right)
$$

This follows statistically as a consequence of the reduced power of the Pearson $r$ relative to the Spearman $p$ when nonmetric or noninterval measurement is given. From the viewpoint of measurement theory the various mathematical manipulations necessary for computation of the parametric product moment have each introduced error, reducing the apparent level of relationship relative to the nonparametric product moment, the 
calculations for which do not introduce this error. In otherwords since the Spearman $p$ treats the values of the variables only as ordinal ranks its value is unaffected by the ordinality of the scales while the strength of Pearson $r$ is dependent upon the scales reaching at least an interval level.

It also follows that, to the extent that the values of $x$ and $y$ approach an interval level of measurement, the error introduced in the computation of the Pearson will be reduced, increasing its power relative to the Spearman.

$$
\text { If, } r_{x y}=2 \sin \left(\frac{\pi}{6} p_{x y}\right) \text {, }
$$

then, at least for this relationship, $x$ and $y$ may be considered to be interval values. Likewise, it follows that $r_{x y}$ approaches the absolute value of one as its limit, then the measure of $x, M(X)$, and the measure of $y, M(y)$, may be considered to be interval for this relationship. This special case corresponds exactly to the criterion of measurement requiring high empirical import which Hempel and Dingle proposed.

The comparatively low values of the $r$ when $x$ and $y$ were not interval may be thought of as a result of scattering around Pearson regression lines, a scattering to which the Spearman is insensitive. Generalization of the above argument beyond a simple linear relationship of $x$ and $y$ would require equation of more sophisticated tests of relatedness, for the value of the Pearson is dependent upon the linearity of the relationship while: the Spearman is powerful only for functions whose absolute values constantly increase. The more general case, though more complicated statistically, is identical in logical structure with the above.

Scales developed by the methods of measurement such as Thurstone's or Guttman's thus may, when related to one another, be tested to find if they reach an "interval equivalence." At times it will undoubtedly be discovered that the numerals given for scale ranks and the score for a Guttman scale may be considered to be numbers. At other times the "equal appearing intervals" of the Thurstone will be found to be effectively equal.

This test for interval equivalence does not test for metric equivalence, since it is insensitive to the zero point. Nevertheless, an interval scale results (as does a metric) in numbers, but an ordinal results only in numerals. As a consequence, statements relating quantities, statements of scientific law form, may be made if it can be assumed that the only difference between metric and interval measurement is in the placement of the zero. Thus, any statement rendered as $\mathrm{x}=\mathrm{y}$ where the two values are of metric level would be rendered as $\mathrm{x}=\mathrm{y}+\mathrm{e}$ when either or both values are interval. 5 
1. See Norman R. Campbe11, Foundations of Science, New York: Dover Publications, 1957, Part II.

2. See James S. Coleman, Introduction to Mathematical Sociology, Glencoe: The Free Press, 1964, Chapter 2.

3. See Carl G. Hempe1, Fundamentals of Concept Formation in Empirical Science, Chicago: University of Chicago Press, 1952, Chapter III.

4. See Herbert Dingle, "A Theory of Measurement," British Journal for the Philosophy of Science, Vol. I, no. 1, May, 1950, pp. 5-26.

5. This formulation has one further practical consequence. If interval equivalence is established among the variables involved, perhaps using a subsample of the total number of persons or cases studied, then it may well be possible that the final analyses can be legitimately based upon parametric instead of non-parametric methods. 